\title{
A precise navigation device for fixation of patella fractures with modified K-wire tension band:a comparative retrospective study
}

Fuming Wang ${ }^{1,2}$, Haolan Xiong ${ }^{3}$, Xiaotao Long ${ }^{2}$, Yang Li $i^{2}$ Xiaohua Chen ${ }^{2}$ and Gang Wang ${ }^{1 *}$

\begin{abstract}
Background: Traditionally, the technique of modified tension band wires (MTBW) has been the most commonly used surgical procedure. The purpose of this study is to design a precise navigation device that can obtain a standard position of K-wires for (MTBW) and to compare the precise MTBW (P-MTBW) by a navigation device with the conventional MTBW (C-MTBW) by hands in a retrospective study.

Methods: The device was designed by solidworks2012 software (USA), which could provide a precise guidance for obtaining parallel K-wires. Besides, it could set the distance between two K-wires and the level of K-wires below patellar anterior surface. From June 2014 to August 2018, a total of 112 patients were employed in this retrospective study. The patients were divided into P-MTBW group and C-MTBW group according to the surgical technique with or without the precise navigation device. We needed to record and analyze the operation time and the number of fluoroscopy, postoperative internal fixation imaging, knee function and complications.

Results: There were 54 patients in P-MTBW group and 58 patients in C-MTBW group. There were statistically significant differences $(P<0.001)$ in the operation time between P-MTBW group (39.5 \pm 4.7 ; range, 32-49 $\mathrm{min})$ and C-MTBW group (53.7 \pm 6.8 ; range, $42-71 \mathrm{~min})$. The number of intraoperative fluoroscopy was significantly less $(P<$ $0.001)$ in P-MTBW group (4.2 \pm 1.4$)$ versus that of C-MTBW group $(8.3 \pm 2.7)$. According to lowa knee score, there was no significant difference ( $P=0.268$ at 1 year) in function between the two groups. According to our own evaluation criteria for MTBW, anyone in the P-MTBW group was excellent and 26 patients were excellent, 20 patients were good, and 2 patients were fair in the C-MTBW group.
\end{abstract}

Conclusion: The navigation device can reduce operation time and intraoperative fluoroscopy frequency. P-MTBW fixation is an accurate and effective surgical procedure for patella fractures.

Keywords: Navigation device, Patella fractures, Modified K-wire tension band

\footnotetext{
* Correspondence: orthoboy@163.com

'Department of Orthopaedics and Traumatology, Nanfang Hospital, Southern Medical University, Guangzhou, Guangdong, People's Republic of China

Full list of author information is available at the end of the article
}

(c) The Author(s). 2021 Open Access This article is licensed under a Creative Commons Attribution 4.0 International License, which permits use, sharing, adaptation, distribution and reproduction in any medium or format, as long as you give appropriate credit to the original author(s) and the source, provide a link to the Creative Commons licence, and indicate if changes were made. The images or other third party material in this article are included in the article's Creative Commons licence, unless indicated otherwise in a credit line to the material. If material is not included in the article's Creative Commons licence and your intended use is not permitted by statutory regulation or exceeds the permitted use, you will need to obtain permission directly from the copyright holder. To view a copy of this licence, visit http://creativecommons.org/licenses/by/4.0/ The Creative Commons Public Domain Dedication waiver (http://creativecommons.org/publicdomain/zero/1.0/) applies to the data made available in this article, unless otherwise stated in a credit line to the data. 


\section{Introduction}

Patella fractures account for approximately $1 \%$ of fractures on adults $[1,2]$. The transverse fracture is the most common type of patella fracture, which often causes functional disability of the knee extensor apparatus with the displacement of the fractured fragments [3].

Traditionally, the technique of modified tension band wires (MTBW) has been the most commonly used surgical procedure and is considered the gold standard for transverse patella fracture [4-7]. This technique consists of two parallel $\mathrm{K}$-wires perpendicular to the fracture line and an eight-shaped wire passing anteriorly over the patella and behind the K-wires. According to the AO principle $[8,9]$, only when the two K-wires remain parallel can convert the tension force of the anterior patella into a compressive force across the articular surface, thus promoting fracture healing. Although the classic MTBW technique is reserved for transverse fractures, certain comminuted fractures can also be treated with a tension band construct if the articular surface is intact enough to allow for compression [10].

Parallel K-wires are the key to the tension band technique, usually depending on the experience and hand feeling of the surgeon. There are no reports about a device that remains two K-wires absolutely parallel; hence, we have designed a precise device to simplify surgery. This device can provide a precise guidance to obtain a parallel $\mathrm{K}$-wires placement in any configuration, which can set the distance between two K-wires and the level of K-wires below patellar anterior surface, thus reducing the operation time and intraoperative fluoroscopy. The goal of this study is to compare the precisely modified tension band wiring (P-MTBW) by a navigation device with the conventionally modified tension band wiring (C-MTBW) by hands in a retrospective study. We hypothesize that the P-MTBW would exhibit a better performance regarding surgical time, intraoperative fluoroscopy, functional score and fewer complications than that of C-MTBW.

\section{Materials and methods}

\section{Design of the guide device (Fig. 1)}

The device was designed by solidworks2012 software (USA). The design process turned to professional engineers, which could meet the functional requirements of the design and fit for industrial production. Two Ushaped arms of the device constitute the main structure through the cross bar c. Sleeves a and b are both divided into inner and outer doubled layer sleeves, while the outer sleeve is fixed on the main structure of the device and the inner sleeve can slide onto the outer sleeve. The centerline of Two U-shaped arms is controlled by the main structure of the device. The plane of the bottom of the tray e is parallel to the plane of the centerline of sleeves a and b, and both bar $\mathrm{c}$ and rod $\mathrm{d}$ have rulers. The tray e touches the front surface of the patella, and the distance between the plane of K-wires and the front surface of the patella is adjusted by sliding rod $\mathrm{d}$. The distance between sleeves $\mathrm{a}$ and $\mathrm{b}$ is adjusted by the cross bar c.

\section{Clinical study}

This study protocol was approved by our hospital Ethics Committee. This retrospective study reviewed patients with patellar fractures who underwent MTBW surgical treatment from June 2014 to August 2018. The inclusion criteria are shown as follows: (1) transverse fractures with or without a single additional fragment; (2) 18 to 65 years without previous knee surgery; (3) the articular displacement is greater than $2 \mathrm{~mm}$ or fragment separation is greater than $3 \mathrm{~mm}$ on radiography; (4) C1 and C2 type with consideration to AO classification; (5) MTBW with or without a navigation device; (6) followup at least 12 months. The exclusion criteria: (1) associated with a fracture of the distal femur or tibial plateau; (2) previous knee diseases such as osteoarthritis. According to these criteria, 112 patients were included in the study. The patients were divided into C-MTBW and PMTBW according to the surgical technique with or

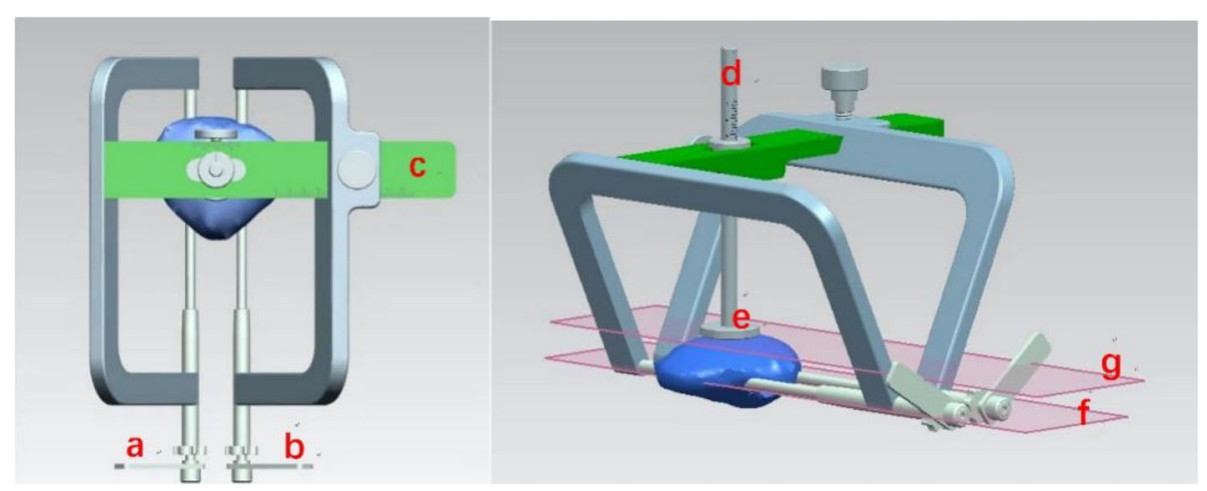

Fig. 1 Design appearance of the navigation device 
without the precise navigation device. There were 58 patients treated with the C-MTBW method and 54 patients treated with P-MTBW by the precise navigation device. We needed to record and analyze the operation time and the number of fluoroscopy, postoperative internal fixation imaging, function, and complications.

All patients underwent the standard MTBW technique. Approach and reduction techniques depend on the standard technique according to the AO principle, while the difference was that the P-MTBW group uses a self-designed precise navigation device to guide the $\mathrm{K}$ wires. Adjusting the device parameters according to the width and thickness of patella measured before surgery. The distance of K-wires was set at one third of the widest diameter of the patella, and the level of the K-wires was set according to the thickness of the outer third of the patella. After setting the parameters of device, Kwire was implanted through the sleeve (Fig. 2).

\section{Postoperative management and evaluation}

For all patients, an elastic bandage was used for $48 \mathrm{~h}$ after surgery for reducing swelling. Isometric quadriceps exercise and straight leg raises were started when pain had subsided. The knee joint was protected by the knee adjustable brace that allows $60^{\circ}$ of motion after surgery, $90^{\circ}$ of motion after 2 weeks and no restriction after 4 weeks. Postoperative follow-ups were arranged for one and 2 weeks, $1,2,3,6$, and 12 months, and the amount duration was longer than 1 year. Common complications included incision infection, failure of internal fixation, fracture displacement, and K-wire irritation. After 1 year of surgery, the knee function was evaluated according to the Iowa knee score criteria.

Postoperative imaging usually was used to evaluate the reduction of the fracture, which ignored the assessment of the internal fixation position. We believe it is necessary to establish a standard evaluation strategy for Kwires of MTBW technique. All patients should have standard X-rays of the knee on two planes after surgery.
An AP viewing and a lateral view are evaluated according to the angle between the K-wires. The angle is measured by two senior doctors respectively. If the error is within $2^{\circ}$, the average value is taken. If the error exceeds $2^{\circ}$, we seek help from the third doctor. If the angle at both views is less than $5^{\circ}$, it is defined as excellent. If the angle at one view is between $5^{\circ}$ and $15^{\circ}$, it is defined as good. If the angle at both views is between $5^{\circ}$ and $15^{\circ}$, it is defined as fair. If the angle at any view is more than $15^{\circ}$, it is bad.

Data were presented as mean \pm SD for continuous variables and the number of categorical variables. To compare two groups, there was a two-sample $t$ test used for continuous variables, Pearson chi-square tested for categorical variables, and Wilcoxon rank sum tested for ordinal categorical variables. The differences were presented as mean (95\% CI) for continuous variables and odds ratio $(95 \% \mathrm{CI})$ for categorical variables. The significance level was set at 0.05 for all the tests, and data was entered and analyzed with SPSS21.0 statistical software (SPSS Inc., Chicago, IL, USA).

\section{Result}

There were 54 patients in $\mathrm{P}$-MTBW group and 58 patients in C-MTBW group, 3 cases in P-MTBW group and 5 cases in C-MTBW group lost to followup. Demographics of both groups were no statistically significant differences (Table 1). There were statistically significant differences $(P<0.001)$ in the operation time between P-MTBW group (39.5 \pm 4.7 ; range, $32-49 \mathrm{~min})$ and C-MTBW group $(53.7 \pm 6.8$; range, $42-71 \mathrm{~min})$. The number of intraoperative fluoroscopy was significantly less $(P<0.001)$ in the P-MTBW group $(4.2 \pm 1.4)$ versus that of C-MTBW group $(8.3 \pm 2.7)$. According to Iowa knee score, there was no significant difference $(P=0.752$ at 1 month, $P=0.836$ at 3 months, $P=0.363$ at 6 months, $P=0.268$ at 1 year) in the function between the two groups (Table 2).

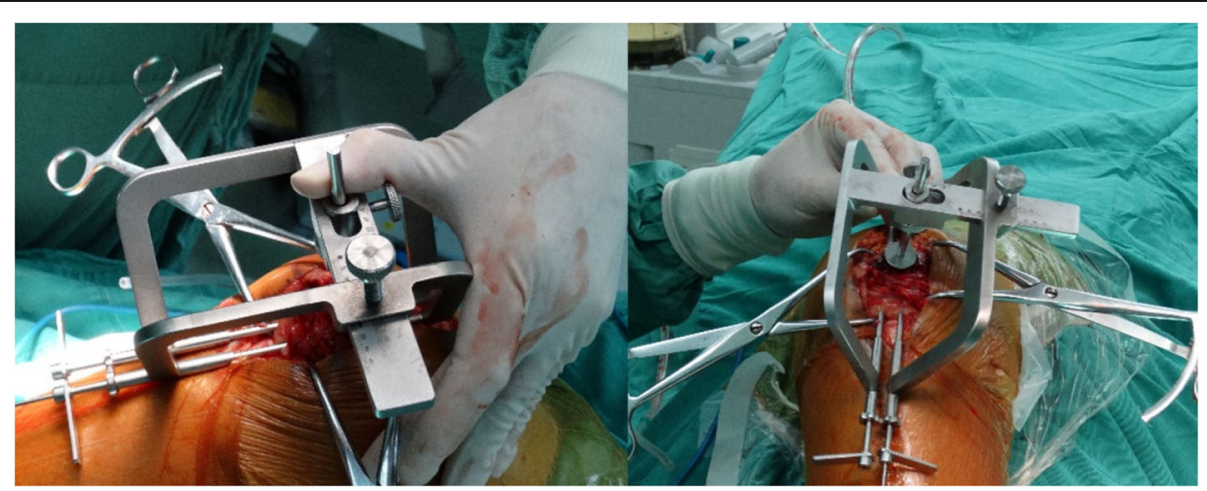

Fig. 2 The physical appearance of the navigation device during the operation 
Table 1 Clinical demographic data between the 2 groups

\begin{tabular}{lllll}
\hline Variables & P-MTBW(54) & C-MTBW(58) & Test statistics & P value \\
\hline Age (years) & $38.5 \pm 12.7$ & $40.5 \pm 12.0$ & & 0.297 \\
Gender & & & 0.735 & 0.391 \\
$\quad$ Male & 35 & 33 & & \\
$\quad$ Female & 19 & 25 & -11.3 & $P<0.01$ \\
Operation time & $39.5 \pm 4.7$ & $53.7 \pm 6.8$ & -10.1 & $P<0.01$ \\
No. of fluoroscopy & $4.2 \pm 1.4$ & $8.3 \pm 2.7$ & 0.101 & 0.751 \\
Complications & 9 & 11 & & \\
\hline
\end{tabular}

According to our own evaluation criteria, all patients in the P-MTBW group were excellent, and 26 patients were excellent, 20 patients were good, and 2 were fair in the C-MTBW group. The comparison between the two groups was statistically significant $(P<0.001)$ (Table 3$)$.

There was no significant difference in the incidence of complications $(P=0.751)$ between the two groups, and none of all patients in this study were infected. One patient experienced failure of internal fixation due to steel wire breakage, five patients had K-wire irritation, and three were presented with fracture displacement in the P-MTBW group. In the C-MTBW group, three patients had internal fixation failure due to wire breakage and $\mathrm{K}$ wire withdrawal, six patients had $\mathrm{K}$-wire irritation, and two presented with fracture displacement. The removal rate of internal fixation was not calculated in this study because of custom.

\section{Discussion}

Although there are new techniques such as cannulated screw tension band $[11,12]$,

cable pin system [13], and patella plate [14], the MTBW is still the most widely accepted surgical method for the treatment of patellar transverse fracture [7]. The MTBW technique is easy to operate on and economical to reduce the burden on patients, and the key of MTBW technique is to keep two K-wires parallel $[8,15]$. The manual operation is not enough to keep two K-wires parallel and needs to be repeatedly adjusted; hence, we design and create this precise guiding device. Besides, it can set the distance between two K-wires and the level of K-wires below patellar anterior surface. According to the anatomic parameters of preoperative measurement, it can set distance accurately in the navigation device. Our study shows that the navigation device is accurate and effective, which can reduce the operation time and intraoperative fluoroscopy frequency and is beneficial to the treatment of patellar transverse fracture.

So far, there is no research on evaluating the quality of MTBW technique; hence, it is necessary to establish an evaluation strategy for K-wires of MTBW technique. The MTBW technique should be evaluated according to the angle between the two K-wires in an AP view and a lateral view after surgery. There is only 45\% (26/58) excellence in C-MTBW group and all are excellent in PMTBW group. The level of the $\mathrm{K}$-wires and the distance between two K-wires are not included in the evaluation strategy, because a complex evaluation strategy is not easy to accept and understand. The evaluation strategy for MTBW technique is highly necessary, so that we can adjust the position of $\mathrm{K}$-wires during the operation.

According to the AO principle [8], the ideal level for the $\mathrm{K}$-wires lies in the center of the patella, approximately $5 \mathrm{~mm}$ below its anterior surface. In practice, the $\mathrm{K}$-wires are closer to the articular than to the anterior surface. Hsu [16] reported the depth of K-wires involving 170 patients treated with MTBW: 37 (22\%) patients at superficial third and $133(78 \%)$ patients at the middle third of the patella and superficially placed Kirschner wires increased the rate of minor loss of reduction.

Table 2 According to lowa knee score

\begin{tabular}{|c|c|c|c|c|c|c|c|c|c|}
\hline \multirow{2}{*}{\multicolumn{2}{|c|}{$\begin{array}{l}\text { Follow-up time } \\
\text { Function }\end{array}$}} & \multicolumn{2}{|c|}{1 month } & \multicolumn{2}{|c|}{3 months } & \multicolumn{2}{|c|}{6 months } & \multicolumn{2}{|l|}{1 year } \\
\hline & & PMTBW & CMTBW & PMTBW & CMTBW & PMTBW & CMTBW & PMTBW & CMTBW \\
\hline \multirow{4}{*}{$\begin{array}{l}\text { lowa } \\
\text { knee } \\
\text { score } \\
\text { criteria }\end{array}$} & $90-100$ & 5 & 4 & 27 & 28 & 35 & 33 & 38 & 35 \\
\hline & 80-89 & 22 & 25 & 14 & 15 & 12 & 14 & 11 & 12 \\
\hline & 70-79 & 19 & 18 & 7 & 5 & 3 & 4 & 2 & 5 \\
\hline & $\leq 69$ & 8 & 11 & 4 & 7 & 1 & 3 & 0 & 1 \\
\hline \multicolumn{2}{|c|}{ Test statistics } & \multicolumn{2}{|l|}{-0.316} & \multicolumn{2}{|l|}{-0.207} & \multicolumn{2}{|l|}{-0.911} & \multicolumn{2}{|l|}{-1.107} \\
\hline \multicolumn{2}{|l|}{$P$ value } & \multicolumn{2}{|l|}{0.752} & \multicolumn{2}{|l|}{0.836} & \multicolumn{2}{|l|}{0.362} & \multicolumn{2}{|l|}{0.268} \\
\hline
\end{tabular}


Table 3 Evaluation of the accuracy of the guide device

\begin{tabular}{lllll}
\hline Variables & & P-MTBW & C-MTBW & Test statistics \\
\hline K-wires & $<5^{\circ}$ in both views & 54 & 26 & -6.408 \\
of MTBW & $5-15^{\circ}$ in one view & 0 & 30 & \\
evaluation & $5-15^{\circ}$ in both views & 0 & 2 & \\
strategy & $>15^{\circ}$ in any view & 0 & 0 & \\
&
\end{tabular}

There is a recently published study by Yang [15] et al. show that the wide distance between them and the K-wires at the deep level might be more helpful for MTBW. Ling [17] performed a finite element analysis, and posteriorly placed K-wires make optimal stability possible. We believe that it should be located $5 \mathrm{~mm}$ below the anterior surface of the lateral third, because the anterior surface of the patella is irregular and the lateral part of the patella is thinner.

There are some limitations to our study. First, it is not a prospective and randomized study. Second, all the operations were open, and the closed reduction and internal fixation guided by the guidance should be further carried out in the later. Finally, we do not distinguish the level of K-wires and the distance between the K-wires; finite element analysis [18, 19] can be carried out for comparison in the future.

In conclusion, the navigation device can reduce the operation time and intraoperative fluoroscopy frequency. PMTBW fixation is an accurate and effective surgical procedure for treatment of transverse patellar fractures.

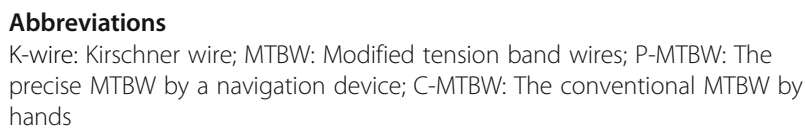

\section{Acknowledgements}

None.

\section{Authors' contributions}

FM and GW are responsible for the whole study. FM and HL wrote the manuscript, and XT, YL, and XH assisted in the surgery. All authors have read and approved the manuscript for submission.

\section{Funding}

This study was sponsored by the Chongqing Yuzhong District Science and Technology Project (No. 20170126).

\section{Availability of data and materials}

All of the data are available in contact with the correspondent author.

\section{Ethics approval and consent to participate}

This study was approved by Ethics Committee of Chongqing General Hospital (Approval No. 20170126).

\section{Consent for publication}

The participant enrolled into the study agreed to the use of data for research.

\section{Competing interests}

The authors declare that they have no competing interests.

\section{Author details}

'Department of Orthopaedics and Traumatology, Nanfang Hospital, Southern Medical University, Guangzhou, Guangdong, People's Republic of China.

${ }^{2}$ Department of Orthopaedics and Traumatology, Chongqing General
Hospital, University of Chinese Academy of Sciences, Chongqing, People's Republic of China. ${ }^{3}$ Department of anesthesiology, Chongqing General Hospital, University of Chinese Academy of Sciences, Chongqing, People's Republic of China.

Received: 30 June 2020 Accepted: 14 January 2021

Published online: 01 February 2021

\section{References}

1. Larsen P, et al. Incidence and epidemiology of patellar fractures. Orthopedics. 2016;39(6):e1154-8.

2. Melvin JS, Mehta S. Patellar fractures in adults. J Am Acad Orthop Surg. 2011;19(4):198-207.

3. Kakazu R, Archdeacon MT. Surgical management of patellar fractures. Orthop Clin North Am. 2016;47(1):77-83.

4. Lotke PA, Ecker ML. Transverse fractures of the patella. Clin Orthop Relat Res. 1981;158:180-4.

5. Weber $\mathrm{MJ}$, et al. Efficacy of various forms of fixation of transverse fractures of the patella. J Bone Joint Surg Am. 1980;62(2):215-20.

6. Schuett DJ, et al. Current treatment strategies for patella fractures. Orthopedics. 2015;38(6):377-84

7. Henrichsen JL, et al. Treatment of patella fractures. Orthopedics. 2018;41(6):e747-55.

8. Buckley RE, Moran CG, Apivatthakakul T. AO principles of fracture management Third Edition. Stuttgart: Thieme; 2017. p. 853-64.

9. Blum L, Hake M. ORIF patella fracture with a tension band construct. Orthop Trauma. 2017;31(Suppl 3):S8-s9.

10. Hambright DS, et al. Revisiting tension band fixation for difficult patellar fractures. J Orthop Trauma. 2017;31(2):e66-72.

11. Tan H, Dai P, Yuan Y. Clinical results of treatment using a modified K-wire tension band versus a cannulated screw tension band in transverse patella fractures: A strobe-compliant retrospective observational study. Medicine (Baltimore). 2016;95(40):e4992.

12. Lin T, et al. Comparison of the outcomes of cannulated screws vs. modified tension band wiring fixation techniques in the management of mildly displaced patellar fractures. BMC Musculoskelet Disord. 2015;16:282.

13. Mao N, et al. Comparison of the cable pin system with conventional open surgery for transverse patella fractures. Clin Orthop Relat Res. 2013;471(7):2361-6.

14. Ellwein A, et al. Outcomes after locked plating of displaced patella fractures: a prospective case series. Int Orthop. 2019;43(12):2807-15.

15. Yang TY, et al. Treatment of displaced transverse fractures of the patella: modified tension band wiring technique with or without augmented circumferential cerclage wire fixation. BMC Musculoskelet Disord. 2018;19(1):167.

16. Hsu KL, et al. Factors affecting the outcomes of modified tension band wiring techniques in transverse patellar fractures. Injury. 2017;48(12):2800-6.

17. Ling $M$, et al. Where should Kirschner wires be placed when fixing patella fracture with modified tension-band wiring? A finite element analysis. J Orthop Surg Res. 2019;14(1):14.

18. Gok K, et al. Biomechanical effects of three different configurations in Salter Harris type 3 distal femoral epiphyseal fractures. J Brazilian Soc Mech Sci Eng. 2016;39(4):1069-77.

19. Inal S, et al. Comparison of the biomechanical effects of pertrochanteric fixator and dynamic hip screw on an intertrochanteric femoral fracture using the finite element method. Int J Med Robot. 2015;11(1):95-103.

\section{Publisher's Note}

Springer Nature remains neutral with regard to jurisdictional claims in published maps and institutional affiliations. 